\title{
Invarianza Factorial de una Escala de Autoeficacia sobre Conductas Académicas en Universitarios Hombres y Mujeres
}

\author{
Judith M. Rodríguez, Martha Ornelas, José R. Blanco y Humberto Blanco* \\ Universidad Autónoma de Chihuahua. Facultad de Ciencias de la Cultura Física. DES Salud CAs 101 y \\ 121. Calle Escorza 900, CP 31000 Chihuahua, Chih.- México. (e-mail: jurodrig@uach.mx, \\ mornelas@uach.mx, jblanco@uach.mx y hblanco@uach.mx) \\ *autor a quien debe ser dirigida la correspondencia.
}

Recibido Mar. 6, 2015; Aceptado May. 14, 2015; Versión final Jul. 5, 2015, Publicado Dic. 2015

\begin{abstract}
Resumen
El presente estudio analiza las propiedades psicométricas de la escala autoeficacia en conductas académicas en hombres y mujeres universitarios. La muestra total fue de 1545 participantes; 616 mujeres y 929 hombres, con una edad media de 18.11 años y 18.27 años respectivamente. Los análisis factorial confirmatorios mostraron que una estructura trifactorial (atención, comunicación y excelencia) es viable y adecuada para ambas poblaciones, mujeres y hombres, de acuerdo a los requisitos psicométricos establecidos cuando los informantes son los propios alumnos. Además, la estructura factorial, las cargas factoriales y los interceptos se consideran invariantes en las dos poblaciones. Sin embargo, existen diferencias entre las poblaciones para las medias de los factores atención y excelencia.
\end{abstract}

\section{Factorial Invariance of a Self-Efficacy Scale on Academic Behavior of University Students, Men and Women}

\begin{abstract}
The present study analyses the psychometric properties of the a self-efficacy scale on academic behavior in university students, men and women. The overall sample consisted of 1545 subjects: 616 women and 929 men, with a mean age of 18.11 years and 18.27 years, respectively. Psychometric analysis showed that a three-factorial structure (attention, communication and excellence) was viable and adequate for both populations, men and woman, according to the established psychometric requirements when the informers are the students themselves. The results showed that factor structure, factor loadings and intercepts of the instrument could be considered invariant across groups. However, there are differences between groups for the means of the factors attention and excellence.
\end{abstract}

Keywords: self-efficacy; factor structure; measurement invariance; multigroup confirmatory factor analysis 


\section{INTRODUCCIÓN}

Un ideal común a todas las personas es conseguir determinadas metas, la propia motivación impulsa al ser humano a emprender conductas específicas en función de los logros que éste pretende alcanzar. Mas no basta conocer con claridad aquello que deseamos lograr, ni tampoco el mejor medio para poder conseguirlo; es preciso juzgarse capaz de utilizar las capacidades y las habilidades personales ante circunstancias muy diversas. La percepción de las personas acerca de su propia eficacia se alza como un requisito fundamental para desarrollar con éxito las acciones conducentes al logro de los objetivos personales. Dicha autopercepción, denominada autoeficacia, ejerce una profunda influencia en la elección de tareas y actividades, en el esfuerzo y en la perseverancia de las personas cuando se enfrentan a determinados retos e incluso en las reacciones emocionales que experimentan ante situaciones difíciles (León-Rubio et al., 2011; Wolters, 2004).

En el área de la psicología educativa, el constructo de autoeficacia ha recibido especial atención y se han generado importantes avances de investigación que han contribuido al mejoramiento de prácticas pedagógicas y de enseñanza (Bandura, 2006). La investigación empírica ha demostrado, de manera amplia, que la autoeficacia resulta ser más predictiva del rendimiento académico que otras variables cognitivas (Bandura, 1982; Schmidt et al., 2008). En definitiva, las creencias de autoeficacia representan un mecanismo cognitivo que media entre el conocimiento y la acción y que determina, junto con otras variables, el éxito de las propias acciones (Sansinenea et al., 2008; Zimmerman y Kitsantas, 2005).

La falta de motivación en los estudiantes es una de las preocupaciones más importantes de la mayoría de los profesores. Casi todos los proyectos de investigación relacionados con la motivación, que incluyen estructuras relacionadas con las creencias de los estudiantes acerca de sus habilidades para llevar a cabo sus tareas académicas, afirman que estas creencias han sido ignoradas en el desarrollo de patrones de motivación (Hasheminasab et al., 2014). Una forma de conceptualizar las creencias de los estudiantes sobre sus capacidades para llevar a cabo sus tareas escolares que ha sido utilizado por los investigadores de la motivación es la autoeficacia; se ha demostrado que niveles altos de autoeficacia conducen a un mejor rendimiento en tareas académicas (Bandura, 1997; Javanmard et al., 2012).

Las creencias de autoeficacia determinan cómo la gente se siente, piensa, se motivan y se comportan. Tales creencias producen estos efectos a través de cuatro procesos principales: cognitivos, motivacionales, afectivos y de selección. Un fuerte sentido de eficacia aumenta la percepción de realización y de bienestar personal de muchas maneras. Las personas con alta confianza en sus capacidades perciben las tareas difíciles como desafíos a ser superados y no como amenazas que deben evitarse. Tal perspectiva eficaz fomenta el interés intrínseco y ensimismamiento profundo en las actividades (Bandura, 1982). La autoeficacia, también llamada capacidad percibida, se refiere pues a la confianza que la gente tiene en sus habilidades para el éxito en una tarea determinada (Bandura, 1997; Shkullaku, 2013). La autoeficacia se explica en el marco teórico de la teoría cognitiva social de Bandura, quien declaró que los logros humanos depende de las interacciones entre los propios comportamientos, los factores personales y las condiciones ambientales (Bandura, 1982; Mahyuddin et al., 2006). Según la teoría cognitiva social, la autoeficacia es una de las variables más importantes que influyen en el rendimiento académico puesto que las personas pueden realizar mal en tareas no necesariamente porque carecen de la capacidad de tener éxito, sino porque carecen de confianza en sus capacidades (Robbins et al., 2004; Shkullaku, 2013).

El presente trabajo, clasificado por Montero y León (2005) como estudio instrumental, se ha dirigido a proporcionar apoyo empírico a la división factorial de la Escala Autoeficacia en Conductas Académicas; lo que se justifica por la importancia de comprobar la estructura factorial de un instrumento y la equivalencia psicométrica del mismo en distintos grupos; ya que en el contexto de la comparación intergrupal, es indispensable plantearse la necesidad de llevar a cabo la adaptación de un instrumento de medida psicológica que cumpla con todos los criterios de equivalencia, pero sobre todo plantearse si la misma estructura factorial es aplicable a distintos grupos de sujetos 0 , de modo más genérico, a distintas poblaciones (Abalo et al., 2006; Arbuckle, 2012). Por tanto, este trabajo pretende, por una parte, indagar si se replican los resultados psicométricos propuestos por Blanco et al. (2011) para la Escala Autoeficacia en Conductas Académicas (EACA) y, por otra, ampliarlos. Para ello, en primer lugar se comprobará el grado de congruencia de la estructura factorial del EACA obtenida en el presente estudio y la reportada por (Blanco et al., 2011). En segundo lugar, se calculará la invarianza factorial entre las muestras del presente estudio.

\section{METODOLOGÍA}

La metodología seguida en este trabajo se describe en cuatro subsecciones: participantes, instrumento, procedimiento y análisis de datos 


\section{Participantes}

La muestra de 1545 participantes, 616 (39.1\%) mujeres y 929 (60.1\%) hombres, se obtuvo mediante un muestreo por conveniencia, tratando de abarcar la representatividad de las diferentes licenciaturas que se ofrecen en la Universidad Autónoma de Chihuahua. Las edades de las mujeres fluctúan entre los 17 y 20 años, con una media de 18.11 y una desviación estándar de 0.69 años; y las de los hombres fluctúan entre los 17 y 20 años, con una media de 18.27 y una desviación estándar de 0.75 años.

\section{Instrumento}

Escala Autoeficacia en Conductas Académicas (EACA) es una encuesta tipo Likert, asistida por computadora de 13 ítems (Blanco et al., 2011) donde el encuestado responde, en una escala de 0 a 10, que tan capaz se siente en cada uno de los ítems de los factores de la escala (Atención, Comunicación y Excelencia).

\section{Procedimiento}

Se invitó a participar en el estudio a los estudiantes de primer ingreso de las licenciaturas que se ofrecen en la Universidad Autónoma de Chihuahua; los que aceptaron participar firmaron la carta de aceptación correspondiente. Luego se aplicó el instrumento antes descrito por medio de una computadora personal utilizando el módulo administrador del instrumento del editor de escalas versión 2.0 (Blanco et al., 2013), en una sesión de aproximadamente 25 minutos; en los centros de cómputo de las unidades académicas participantes. Al inicio de cada sesión se hizo una pequeña introducción sobre la importancia de la investigación y de cómo acceder al instrumento; las instrucciones de como responder se encontraban en las primeras pantallas; antes del primer reactivo del instrumento. Al término de la sesión se les agradeció su participación. Una vez aplicado el instrumento se procedió a recopilar los resultados por medio del módulo generador de resultados del editor de escalas versión 2.0 (Blanco et al., 2013)).

\section{Análisis de datos}

El análisis psicométrico se realizó en dos etapas: 1) análisis factorial confirmatorio y 2) análisis de invarianza factorial; con el fin de obtener una prueba que presente las mejores propiedades para la conformación de los puntajes de autoeficacia en conductas académicas en universitarios mujeres y hombres. Para conducir el análisis factorial confirmatorio para cada muestra, se utilizó el software AMOS 21 (Arbuckle, 2012), las varianzas de los términos de error fueron especificados como parámetros libres, en cada variable latente (factor) se fijó uno de los coeficientes estructurales asociados a uno, para que su escala sea igual a la de una de las variables superficiales (ítems). El método de estimación empleado fue el de Máxima Verosimilitud; siguiendo la recomendación de Thompson (2004), en el sentido de que cuando se emplea análisis factorial confirmatorio se debe corroborar no sólo el ajuste de un modelo teórico sino que es recomendable comparar los índices de ajuste de varios modelos alternativos para seleccionar el mejor.

Para evaluar el ajuste del modelo se emplearon el estadístico Chi-cuadrado, el índice de bondad de ajuste (GFI) y el error cuadrático medio de aproximación (RMSEA) como medidas absolutas de ajuste. El índice de bondad ajustado (AGFI), el Índice Tucker-Lewis (TLI) y el índice de bondad de ajuste comparativo (CFI) como medidas de ajuste incremental. El índice de ajuste normado de parsimonia (PNFI), el índice de calidad de ajuste de parsimonia (PGFI), la razón de Chi-cuadrado sobre los grados de libertad (CMIN/GL) y el Criterio de Información de Akaike (AIC) como medidas de ajuste de parsimonia (Gelabert et al., 2011). Por último, se llevó a cabo un análisis de la invarianza factorial de los modelos de medida obtenidos, siguiendo las recomendaciones de Abalo et al. (2006), y se calculó la fiabilidad de cada una de las dimensiones a través del Alfa de Cronbach y el Coeficiente Omega (Revelle y Zinbarg, 2009).

\section{RESULTADOS}

\section{Análisis factorial confirmatorio}

De acuerdo a los resultados de la Tabla 1 el análisis factorial confirmatorio de 13 ítems agrupados en tres factores en la muestra de mujeres es óptimo (GFI .965 y RMSEA .049) y de acuerdo a las medidas de ajuste incremental y de parsimonia significativamente superior al modelo independiente y muy similar al modelo saturado.

Por otro lado, el análisis factorial confirmatorio en la muestra de hombres (Tabla 1), indica que el modelo de medición de tres factores es aceptable (GFI .968 y RMSEA .051) y de acuerdo a las medidas de ajuste incremental y de parsimonia significativamente superior al modelo independiente y muy similar al modelo 
saturado. En la Tabla 1, $p<.05$; GFI es el índice de bondad de ajuste; RMSEA es el error cuadrático medio de aproximación; AGFI es el índice de bondad ajustado; TLI es el índice Tucker-Lewis; CFI es el índice de bondad de ajuste comparativo; CMIN/DF es la razón de Chi-cuadrado sobre los grados de libertad, y AIC es el criterio de información de Akaike. De acuerdo a los resultados de la Tabla 2, en ambas muestras, todos los ítems saturan adecuadamente en su dimensión (factor) prevista. Observándose intercorrelaciones moderadas entre los factores evidenciando una adecuada validez discriminante.

Tabla 1: Índices absolutos, incrementales y de parsimonia para los modelos generados. Análisis factorial confirmatorio para mujeres y hombres.

\begin{tabular}{|c|c|c|c|c|c|c|c|c|c|}
\hline & Índices absolutos & \multicolumn{2}{c|}{ Índices incrementales } & \multicolumn{2}{c|}{ Índices de parsimonia } \\
\hline Modelo & $\chi^{2}$ & GFI & RMSEA & AGFI & TLI & CFI & CMIN/DF & AIC \\
\hline \multicolumn{8}{|c|}{ Solución factorial para los mujeres } \\
\hline Independiente & $2781.616^{*}$ & 0.321 & 0.237 & 0.321 & 0.000 & 0.000 & 36.662 & 2807.616 \\
\hline Saturado & 0.000 & 1.000 & & & & 1.000 & & 182.00 \\
\hline 3 factores & $147.026^{*}$ & 0.965 & 0.049 & 0.945 & 0.957 & 0.967 & 2.492 & 211.026 \\
\hline \multicolumn{8}{|c|}{ Solución factorial para las hombres } \\
\hline Independiente & $4283.037^{*}$ & 0.405 & 0.241 & 0.306 & 0.000 & 0.000 & 54.911 & 263.634 \\
\hline Saturado & 0.000 & 1.000 & & & & 1.000 & & 182.000 \\
\hline 3 factores & $199.634^{*}$ & 0.968 & 0.051 & 0.950 & 0.956 & 0.967 & 3.384 & 263.634 \\
\hline
\end{tabular}

Tabla 2: Soluciones estandarizadas para el análisis factorial confirmatorio en ambas muestras. En la Tabla, Factor 1 = Atención; Factor $=2$ Excelencia; Factor 3 = Comunicación

\begin{tabular}{|c|c|c|c|c|c|c|}
\hline \multicolumn{7}{|c|}{ Pesos factoriales } \\
\hline & \multicolumn{2}{|c|}{ Factor 1} & \multicolumn{2}{|c|}{ Factor 2} & \multicolumn{2}{|c|}{ Factor 3} \\
\hline Ítem & mujeres & hombres & mujeres & hombres & mujeres & hombres \\
\hline $\begin{array}{l}7 \text { Pongo atención cuando los profesores } \\
\text { dan la clase }\end{array}$ & 0.74 & 0.73 & & & & \\
\hline $\begin{array}{l}13 \text { Escucho con atención las preguntas y } \\
\text { comentarios de mis profesores }\end{array}$ & 0.73 & 0.74 & & & & \\
\hline $\begin{array}{l}2 \text { Escucho con atención cuando el profesor } \\
\text { aclara una duda a un compañero }\end{array}$ & 0.66 & 0.66 & & & & \\
\hline $\begin{array}{l}3 \text { Escucho con atención las preguntas y } \\
\text { aportaciones de mis compañeros }\end{array}$ & 0.64 & 0.62 & & & & \\
\hline $\begin{array}{l}8 \text { Pongo atención cuando un compañero } \\
\text { expone en clase }\end{array}$ & 0.58 & 0.65 & & & & \\
\hline $\begin{array}{l}11 \text { Entrego puntualmente los trabajos que se } \\
\text { me encargan }\end{array}$ & & & 0.73 & 0.73 & & \\
\hline 1 Cumplo con las tareas que se me asignan & & & 0.69 & 0.80 & & \\
\hline $\begin{array}{l}10 \text { Me preparo para mis exámenes } \\
\text { apoyándome en los apuntes de clase, el } \\
\text { texto del curso y lecturas adicionales }\end{array}$ & & & 0.64 & 0.63 & & \\
\hline 12 Soy cumplido en cuanto a mi asistencia & & & 0.63 & 0.56 & & \\
\hline 4 Expreso mis ideas con claridad & & & & & 0.82 & 0.79 \\
\hline $\begin{array}{l}5 \text { Hago comentarios y aportaciones } \\
\text { pertinentes }\end{array}$ & & & & & 0.72 & 0.77 \\
\hline $\begin{array}{l}9 \text { En caso de desacuerdo soy capaz de } \\
\text { entablar un diálogo con mis profesores }\end{array}$ & & & & & 0.68 & 0.65 \\
\hline $\begin{array}{l}6 \text { Me siento bien con mi propio desempeño } \\
\text { cuando hablo enfrente de una clase o } \\
\text { grupo de gente }\end{array}$ & & & & & 0.63 & 0.64 \\
\hline \multicolumn{7}{|c|}{ Correlaciones entre factores } \\
\hline Factor 1 & - & - & & & & \\
\hline Factor 2 & 0.69 & 0.70 & - & - & & \\
\hline Factor 3 & 0.50 & 0.55 & 0.42 & 0.47 & - & - \\
\hline
\end{tabular}




\section{Invarianza de la estructura factorial entre mujeres y hombres universitarios}

Los índices de ajuste obtenidos (Tabla 3) permiten aceptar la equivalencia de los modelos de medida básicos entre las dos muestras. Aunque el valor de Chi-cuadrado excede al exigido para aceptar la hipótesis de invarianza, el resto de índices contradicen esta conclusión (GFI .966; CFI .967; RMSEA .035; AIC 474.666) lo que nos permite aceptar el modelo base de la invarianza (modelo sin restricciones).

Añadiendo al modelo base restricciones sobre las cargas factoriales caracterizamos la invarianza métrica. Los valores que se recogen en la tabla 3 permiten aceptar este nivel de invarianza. El índice de ajuste general $(\mathrm{GFI}=.964)$ y el error cuadrático medio de aproximación $(\mathrm{RMSEA}=.035)$ siguen aportando información convergente en esta dirección. Además, el criterio de información de Akaike $(\mathrm{AIC}=480.403)$ y el índice comparativo de Bentler $(\mathrm{CFI}=.964)$ no sufren grandes variaciones respecto al modelo anterior. Haciendo uso del criterio para la evaluación de los modelos anidados propuesto por Cheung y Rensvold (2002), quiénes sugieren que si el cálculo de la diferencia de los CFI de ambos modelos anidados disminuye en .01 o menos, se da por bueno el modelo restringido y por tanto el cumplimiento de la invarianza factorial; la diferencia entre CFIs obtenida permite aceptar el modelo de invarianza métrica. Podemos concluir hasta ahora que las cargas factoriales son equivalentes en las dos muestras. En la Tabla, p < .05; GFI es el índice de bondad de ajuste; NFI es el índice de ajuste normado; CFI es el índice de bondad de ajuste comparativo; RMSEA es el error cuadrático medio de aproximación y AIC es el criterio de información de Akaike.

Una vez demostrada la invarianza métrica entre las muestras, pasamos a evaluar la equivalencia entre interceptos (invarianza factorial fuerte). Los índices (Tabla 3) muestran un buen ajuste de este modelo, tanto evaluado de modo independiente como analizándolo respecto a su anidamiento con el modelo de invarianza métrica. La diferencia entre los índices comparativos de Bentler es de 0.003; el índice de ajuste general es 0.962 y el error cuadrático medio de aproximación es 0.035 . Aceptada la invarianza fuerte, los dos modelos evaluados son equivalentes respecto a los coeficientes factoriales y a los interceptos.

Tabla 3: Índices de bondad de ajuste de cada uno de los modelos puestos a prueba en la invarianza factorial.

\begin{tabular}{|l|c|c|c|c|c|c|c|}
\hline \multicolumn{1}{|c|}{ Modelo } & \multicolumn{7}{c|}{ Índice de Ajuste } \\
\hline & $\chi^{2}$ & gl & GFI & NFI & CFI & RMSEA & AIC \\
\hline Modelo sin restricciones & $346.666^{*}$ & 118 & 0.966 & 0.951 & 0.967 & 0.035 & 474.666 \\
\hline Invarianza métrica & $372.403^{*}$ & 128 & 0.964 & 0.947 & 0.965 & 0.035 & 480.403 \\
\hline Invarianza factorial fuerte & $393.233^{*}$ & 134 & 0.962 & 0.944 & 0.962 & 0.035 & 489.233 \\
\hline
\end{tabular}

Los factores obtenidos en los análisis factoriales confirmatorios alcanzan valores de consistencia interna por encima de .75 en ambas muestras (hombres y mujeres); evidenciando una consistencia interna adecuada para este tipo de subescalas, particularmente si se considera el número reducido de ítems (Tabla 4).

Tabla 4: Coeficiente omega y alfa para los factores obtenidos

\begin{tabular}{|l|c|c|c|c|}
\hline & \multicolumn{2}{|c|}{ Mujeres } & \multicolumn{2}{c|}{ Hombres } \\
\hline \multicolumn{1}{|c|}{ Factor } & $\Omega$ & - & $\Omega$ & - \\
\hline 1. Atención & 0.803 & 0.797 & 0.811 & 0.804 \\
\hline 2. Excelencia & 0.768 & 0.762 & 0.777 & 0.768 \\
\hline 3. Comunicación & 0.806 & 0.795 & 0.806 & 0.798 \\
\hline
\end{tabular}

Contrastes de las medias de los factores entre mujeres y hombres

Una vez comprobada la invarianza factorial, las diferencias entre las medias de los factores de los dos grupos se estimaron tomando como referente la muestra de hombres, fijando en 0 el valor de las medias para dicha muestra y estimando libremente el valor de las medias para la muestra de mujeres. Las restricciones sobre los coeficientes de regresión e interceptos, requeridos para los contrastes entre las medias se realizaron automáticamente mediante el software AMOS 21 (Arbuckle, 2012). Los resultados de las comparaciones entre medias indicaron que la media de los factores Atención y Excelencia fueron significativamente mayores $(0.177, p<0.001$ y $0.426, p<0.001$ respectivamente) en las mujeres; y sin diferencia en el factor Comunicación. 


\section{DISCUSIÓN FINAL}

En síntesis, el análisis de las propiedades psicométricas, ha mostrado que una estructura trifactorial es viable y apropiada de acuerdo a los requisitos psicométricos establecidos cuando los informantes son los propios alumnos. La estructura de tres factores, atendiendo a criterios estadísticos y sustantivos, ha mostrado adecuados indicadores de ajuste, de fiabilidad y de validez. Sin embargo, consideramos que más estudios son necesarios con el fin de corroborar o refutar los datos obtenidos en la presente investigación.

Dos limitaciones están presentes en este trabajo. La primera es que los participantes son solo estudiantes universitarios mexicanos, lo que supone una amenaza para la posibilidad de generalizar estos resultados. Ampliar la muestra (agregando por ejemplo adultos jóvenes que no son estudiantes) es un área de trabajo de cara al futuro. La segunda limitación proviene del propio instrumento de evaluación, que se basa en el autoinforme y que puede contener los sesgos que se derivan de la deseabilidad social.

\section{CONCLUSIONES}

De los resultados mostrados, de su análisis y de su discusión, y tomando en cuenta que el objetivo principal de este estudio fue el de examinar la estructura factorial y la medición de la invariancia de dicha estructura en alumnos y alumnas universitarios, se pueden obtener las siguientes conclusiones:

1) El Análisis Factorial Confirmatorio, en ambas muestras, indicó que el ajuste de los datos al modelo teórico de 13 ítems agrupados en tres factores es aceptable. Al mismo tiempo que los dos factores así obtenidos presentan en general saturaciones factoriales estandarizadas adecuadas. Por su parte los factores correlacionan entre sí de forma positiva y estadísticamente significativa lo cual muestra que a medida que aumenta la autoeficacia percibida en alguno de los factores, también aumenta en el otro.

2) Los factores en ambas muestras evidenciaron una consistencia interna adecuada, particularmente si se considera el número reducido de ítems en cada uno de ellos.

3) Conjuntamente con todo lo antes dicho, los resultados del análisis de la invarianza factorial entre las muestras; indican una alta congruencia entre pares de factores. Lo que sugiere la existencia de fuertes evidencias de la validación cruzada de la medida y por tanto de la estabilidad de la estructura, hasta que no se demuestre lo contrario.

4) Las comparaciones entre los grupos reflejaron diferencias significativas, a favor de las mujeres, en las medias de los factores Atención y Excelencia. Lo que parece indicar que las mujeres se perciben un poco más autoeficaces que los hombres en relación a dichos factores.

\section{REFERENCIAS}

Abalo J., Lévy J., Rial A., Varela J., Invarianza factorial con muestras múltiples, In Modelización con Estructuras de Covarianzas en Ciencias Sociales by J. Lévy, pp 259-278 Netbiblo, Madrid, (2006)

Arbuckle J. R., AMOS users guide version 21.0, Marketing Department, SPSS Incorporated, Chicago, IL, (2012)

Bandura A., Self-efficacy mechanism inhuman agency, American Psychologist, 37(2), 122-147, (1982)

Bandura A., Self-efficacy: The exercise of Control, Freeman, New York, (1997)

Bandura A., Guide for constructing self-efficacy scales, In Self-efficacy beliefs of adolescents, by F. Pajares, Urdan T. C., pp 307-337 Age Publishing, Inc., Greenwich, (2006)

Blanco H., Martínez M., Zueck M. d. C., Gastélum G., Análisis psicométrico de la escala autoeficacia en conductas académicas en universitarios de primer ingreso, Actualidades Investigativas en Educación, 11(3), 1-27, (2011)

Blanco H., Ornelas M., Tristán J. L., Cocca A., Mayorga-Vega D., López-Walle J., et al., Editor for creating and applying computerise surveys, doi: http://dx.doi.org/10.1016/j.sbspro.2013.12.105, Procedia Social and Behavioral Sciences, 106, 935-940, (2013)

Cheung G. W., Rensvold R. B., Evaluating goodness-of-fit indexes for testing measurement invariance, doi: 10.1207/s15328007SEM0902_5, Structural Equation Modeling, 9(2), 233-255, (2002) 
Gelabert E., García-Esteve L., Martín-Santos R., Gutiérrez F., Torres A., Subirà S., Psychometric properties of the Spanish version of the Frost Multidimensional Perfectionism Scale in women, Psicothema, 23(1), 133139, (2011)

Hasheminasab M., Ghanbari Z., Azizi J. y Shamsi M., Investigating the Relationship between Self-Efficacy with Academic Achievement, Discipline, Urban-Rural and Order Birth of High School Students in Rafsanjan, International Journal of Psychology and Behavioral Research, 3(4), 258-264, (2014)

Javanmard A., Hoshmandja M. y Ahmadzade L., Investigating the relationship between self-efficacy, Cognitive and metacognitive strategies, and academic self-handicapping with academic achievement in male high school students in the Tribes of Fars Province, Journal of life Science and Biomedicine, 3(1), 27-34, (2012)

León-Rubio J. M., Cantero F. J., León-Pérez J. M., Diferencias del rol desempeñado por la autoeficacia en el burnout percibido por el personal universitario en función de las condiciones de trabajo, Anales de Psicología, 27(2), 518-526, (2011)

Mahyuddin R., Elias H., Cheong L. S., Muhamad M. F., Noordin N. y Abdullah M. C., The relationship between students self efficacy and their enghlish language achievement, Jurnal Pendidik dan Pendidikan, 21, 61-71, (2006)

Montero I., León O., Sistema de clasificación del método en los informes de investigación en Psicología, International Journal of Clinical and Health Psychology, 5, 115-127, (2005)

Revelle W., Zinbarg R. E., Coefficients alpha, beta, omega and the glb: comments on Sijtsma, doi: 10.1007/s11336-008-9102-z, Psychometrika, 74(1), 145-154, (2009)

Robbins S. B., Lauver K., Le H., Davis D., Langley R. y Carlstrom A., Do psychosocial and study skill factors predict college outcomes? A meta-analysis, doi: 10.1037/0033-2909.130.2.261, Psychological Bulletin, 130(2), 261-288, (2004)

Sansinenea E., Gil L., Agirrezabal A., Larrañaga M., Ortiz G., Valencia J. F., et al., Autoconcordancia y autoeficacia en los objetivos personales: ¿Cuál es su aportación al bienestar?, Anales de Psicología, 24(1), 121-128, (2008)

Schmidt V., Messoulam N., Molina F., Autoconcepto académico en adolescentes de escuelas medias: presentación de un instrumento para su evaluación, Revista Iberoamericana de Diagnóstico y Evaluación Psicológica, 1(25), 81-106, (2008)

Shkullaku R., The relationship between self - efficacy and academic performance in the context of gender among Albanian students, European Academic Research, 1(4), 467-478, (2013)

Thompson B., Exploratory and Confirmatory Factor Analysis. Understanding concepts and applications. , American Psychological Association, Washington, D C, (2004)

Wolters C., Advancing achievement goal theory: using goal structures and goal orientations to predict students'motivation, cognition and achievement, Journal of Educational Psychology, 96(2), 236-250, (2004)

Zimmerman B., Kitsantas A., Homework practice and academic achievement. The mediating role of selfefficacy and perceived responsibility beliefs, Contemporary Educational Psychology, 30(4), 397-417, (2005) 
